\title{
Improving the Accuracy of Face Annotation in Social Network
}

\author{
C. Jayaramulu \\ Research Scholar \\ Dayananda Sagar University, Bangalore
}

\author{
Sateesh T. K., PhD \\ Chairman and Professor \\ Dayananda Sagar University, Bangalore
}

\begin{abstract}
The across the board utilization of digital cameras and cell telephones, and the prevalence of online photograph sharing applications, for example, "Flickr" and "Facebook" has prompted the production of various accumulations of individual photographs. These accumulations of individual photographs should be overseen by clients. Accordingly, an in number interest exists for programmed substance annotation procedures that encourage proficient and viable pursuit in accumulations of individual photographs. Individual photographs are generally commented along the "who," "where," and "when" measurement in a specific order of significance. In reality, late client studies report that individuals want to arrange their photographs as indicated by who shows up in their photographs (e.g., relatives or companions). The fundamental pointof this paper is to maintain a strategic distance from the duplication ofnames by utilizing random walk (RW) with restarts based semi-directed learning procedure. Utilizing Random walk, this framework re-positions the competitor annotations, in which both the web data and certainty scores of unique annotations are fortified. RW understands the general substance based face annotationissue utilizing the inquiry based strategy. By evacuating the duplication of names, the marking quality will be expanded to more noteworthy degree by which the annotation method will get exact results.
\end{abstract}

\section{Keywords}

Face annotation, content-based image retrieval, machine learning, label refinement, web facial images, weak label.

\section{INTRODUCTION}

Built up face annotation approaches are routinely viewed as an enlarged face affirmation issue. Regulated the propelled media devices are growing so the unmistakable person to person communication gadgets used for sharing photos. The tremendous number of human facial pictures shared over the unmistakable social certifiable application some of this photos are marked really yet enormous quantities of pictures are not named fittingly so the facial annotation are came. The major objective of request based annotation is to consign name to facial picture. To redesign the name way of facial pictures naturally. Facial annotation in like manner joined in highlight zone to recognize the person who appeared in highlight. The model base annotation has more constrainments i.e. it is extra long and all the more radical to assemble enormous measure of human marked get ready facial picture. It is all the more difficult to enhance the models when new persons are incorporated, in which retraining methodology is obliged and last the annotation execution is get the opportunity to be poor when the amount of persons is more. To address the challenges "Auto face annotation" is vital technique which normally gives name of related person. This method is more profitable to various certifiable application for (e.g. Facebook) which illuminates photos exchanged by the customers for managing online gathering and chases the photographs. The interest based face annotation perfect model intends to handle the robotized face annotation task by abusing substance based picture recuperation (CBIR) techniques in mining number of desolately named facial pictures on the web. The essential focus of chase based face annotation is to consign right name marks to a given inquiry facial picture. One test went up against by such SBFA standard is the way by which to suitably mishandle the most near facial pictures and their weak names for the face name annotation task. The fundamental social event of related work is on the purposes of face affirmation and check [5]. The built up investigation that has been extensively considered [6].

The restrictions are (i) eminent marked face pictures assembled in all around controlled circumstances and (ii) incorporate another individual or new get ready data is nontrivial. The second assembling is about the examinations of insipid picture annotation [7] .Train face gathering models by altering the present face affirmation routines. Persevere through the same imperatives above. The third assembling is pivot annotation on individual/family/social photos. In auto picture annotation object affirmation it is hard to accumulate human marked get ready pictures. The Limitations of this method is semantic opening, and (ii) experience the evil impacts of uproarious web data. The fourth assembling is about the examinations of face annotation in mining desolately marked facial pictures on the web [9][10]. A couple of studies consider a human name as the data inquiry, and basically mean to prepare the substance based rundown things by mishandling visual consistency of facial pictures. The web facial picture with the names expelled from its heading. Unsupervised Face Annotation: chasing facial pictures down a given name, clean, and re-rank substance based inquiry things. These works are not in perspective of the request based annotation perfect model. A customized structure that will name persons in individual and get-together photos using face affirmation strategies [11].In Automatic picture naming strategy it uproot the watchword using substance mining method. It just spotlights on substance not on visual components [13]. Sexual introduction Classification taking into record Principle Component Analysis, Linear Component Analysis and Independent Component Analysis, it is watched that ICA and PCA convey less precision in seeing the sex [15]. Interest Based Face Annotation: An effectiveunsupervised name refinement for refining the web facial pictures[2]. For improving the execution they moreover propose progression computation to comprehend broad scale adjusting suitably i.e. bunching based harsh supposition system upgrades the execution of request based face annotation procedure. The effort are one of a kind casingall past work by two things. To light up broad substance based face annotation issue using chase based where face picture as request picture. They unsupervised mark refinement computation which redesigned new name matrix. This effort 
is also related late work of the WRLCC technique [3] it is a united learning arrangement [4].

\section{RELATED WORK}

Assorted studies are perform on face annotation in mining desolately marked facial pictures which are accessible over web in this human name are managed as information request and point is to refine the substance based question things by finishing include facial pictures.

\subsection{Clustering Algorithm with Possibility:}

Model Berg et al. [4] presents the blend of a credibility model with a bunching estimation. This mix is to exhibit the relationship between the facial pictures and the names in their engravings for the facial pictures and the perceived names in the same record. The basically bunching system is used to engraved new pictures and actually association name. For improving the execution grouping method are solidify with credibility model .joining this two strategies there are exact named arranged of appearances. The eventual outcome of this work shows that by examining lingo purposely can make endlessly enhanced bunching besides take in a trademark vernacular classifier to make sense of who is envisioned from substance alone. This framework takes a shot at particular data set we encourage more upgrade with the desire of complimentary substance on website page using essential picture representation and setting model.

\subsection{Graph Based Approach:}

Ozkan and Duygulu [5] proposed an outline based model for finding the densest sub-diagram as the most related result. Proposed a framework to accomplice names and appearances for addressing people in considerable news photo assembling In numerous cases the amount of same faces of addressed individual will be extensive so the faces are dynamically like each other. They proposed the outline based technique to find the equivalent subset with possible course of action of faces with request individual name. Likeness is addressed by SIFT describers. By then apply a greedy outline estimation. Guillaumin et al.[6] familiar a change with combine the constraint that a face is simply depicted once in a photo. There are two circumstances of naming persons in database for finding face of individual and dispensing name to all stands up to The substance based result is not colossally advanced. To improve a contempt outline based procedure show the necessities when streamlining the objective limit .generative models have been proposed to comprehend the multiindividual naming task .by differentiating generative and graph based strategies the most gigantic framework is diagram based method in future extends the graph based system to multi-individual naming Guillaumin et al. [6] proposed to iteratively upgrade the errand in light of a base cost facilitating figuring. In their consequent work Guillaumin et al. [7], they further improve the annotation execution by using partition metric learning techniques to gain perceive highlight in low estimation space.

\subsection{Query Expansion:}

T. Mensink and J.J. Verbeek [8], by using contemplations from inquiry expansion the execution of name-based arrangement can be further gained ground. In this paper they are captivated to finding pictures of people on the web and more clearly named the new pictures. The substance base starting results are not awesome. The displays are depending upon the assumptions. To improve such poor execution proposed "inquiry expansion". They associated this idea on right on time proposed system on which channel the early on result set. Using Gaussian mix exhibiting and logistic discriminant model. The inquiry advancement is improving the execution in both of system. The examination suggest the model picked up from engraving based supervision.

\subsection{Purify Web Facial pictures:}

This arrangements to right disorderly web facial pictures for face affirmation applications [9], [10]. These works are proposed as an essential preprocessing walk in the whole system without grasping propelled methodologies. T.L. Berg, A.C. Berg et al. [9] associated a changed $\mathrm{k}$ means bunching strategy for tidying up the uproarious web facial pictures. Zhao et al. [10] propose system that can learn and see go up against by merging weakly marked substance, picture and highlight. Consistency learning proposed to make face model for unmistakable individual the substance pictures on the web as a fragile indication of congruity and take in dependable face model from gigantic and uproarious get ready sets. Effective and accurate face recognizable proof and taking after is joined. In conclusion key faces are picked by bunching to get minimized and powerful representation. The compellingness is augmentation in view of speak to key face and evacuates duplicate key face. They used the unsupervised machine learning methods and propose a diagram based name refinement figuring to enhance the name quality over the whole recuperation database. Z. Wu, Q. Ke, J. Sun, and H.- Y. Shum [11] principally kept an eye on the face recuperation issue, by using neighborhood and overall components which propose a convincing picture representation. Future Work is to arrange a directed learning figuring to modernize this system to further improve the visual word vocabulary for face. This system is especially versatile, and they orchestrate by using a PC bundle to apply on a web-scale picture database.

\subsection{Retrieval Based Face Annotation:}

D. Wang, S.C.H. Hoi, Y. He. Furthermore, J. Zhu [12] the WLRLCC figuring is revolved around adjusting more segments for the top recouped facial pictures for each inquiry. By fragile name regularized neighborhood bearing coding. Recuperationbased face annotation is usedas a piece of mining tremendous web facial pictures for modified face annotation .there are two challenges first isthe way suitably recoup a huge segment of near facial pictures. Second is the best approach to reasonably perform annotation. They proposed feeble mark regularized adjacent orchestrate coding (WLRLCC) methodology. They moreover proposed the progression computation i.e.WLRLCC count .This figuring helps the execution of the recuperation build face annotation approach as for a sweeping scale web facial picture D. Wang, S.C.H. Hoi, and Y. He et al. [13] this proposed structure investigated a uniting learning arrangement by joining both transductive and inductive learning framework to burrow web facial picturesfor face annotation. They proposed WeaknameLaplacian support vector machine (WL-LapSVM) estimation by grasping WLRLCC computation.

\section{PROPOSED WORK}

This annotation approach consists of following steps as illustrated in fig. 1:

Facial image data collection;

Face detection and facial feature extraction;

High-dimensional facial feature indexing;

Learning to refine weakly labeled data;

Similar face retrieval; 


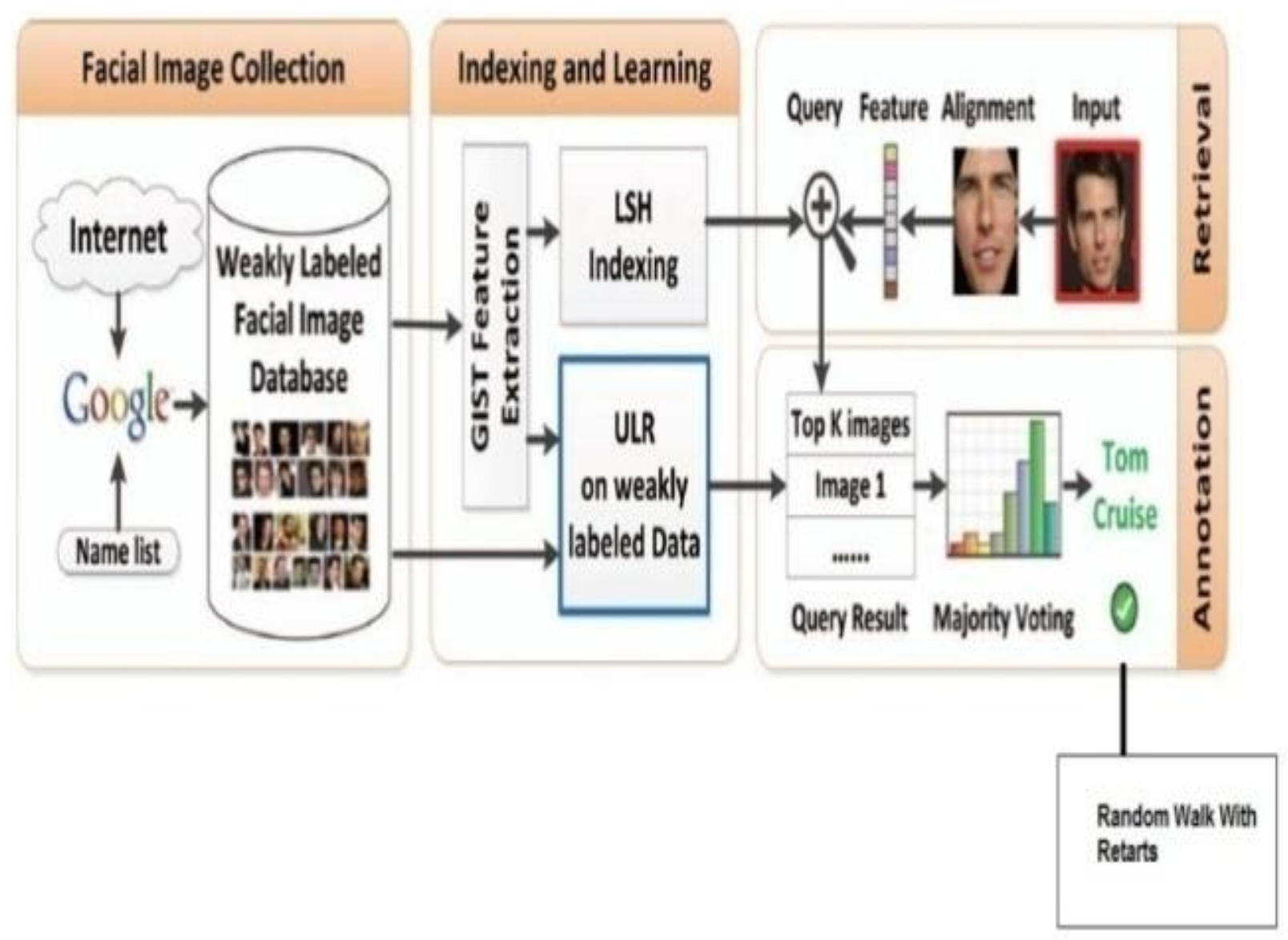

Figure 1:The system flow of the proposed search-based face annotation scheme.

\section{A. Facial Image Data Collection}

In this step we slithered an aggregation of facial pictures from the WWW by a present web crawler (i.e., Google) according to a name list that contains the names of persons to be assembled. As a yield of this method we may get a social event of facial pictures, each of them is associated with some human names. These facial pictures are oftentimes uproarious, which don't by and large contrast with the right human name. In this way, we call such kind of web facial pictures with uproarious names as miserably marked facial picture data.

\section{B. Face Detection And Facial Feature Extraction}

This step is to preprocess web facial pictures to focus facerelated information, including face area and course of action, facial range extraction, and facial segment representation. For face recognizable proof and course of action, we get the unsupervised face game plan framework. For facial part representation, we evacuate the GIST surface segments [2] to identify with the uprooted appearances. In this way, every face can be identified with by a d-dimensional component vector. The engraving based annotation arrangement is only material to the circumstance where both pictures and their engravings are open, and can't be associated with our SBFA structure [1] in view of the nonappearance of complete subtitle information. This Step examinations of sifting web facial pictures, which plans to impact boisterous web facial pictures for face affirmation applications.

\section{High-Dimentional Facial Feature Indexing}

The third step is to list the evacuated parts of the appearances by applying some capable high dimensional indexing framework to energize the endeavor of similar face recuperation in the subsequent step. In our theory, we get the zone fragile hashing (LSH) [3], an astoundingly celebrated and convincing high dimensional indexing system.

\section{Figuring Out How To Refine Weakly Labeled Data}

Other than the indexing step, another key step of the structure is to attract an unsupervised learning arrangement to enhance the name way of the pathetically marked facial pictures. This methodology is crucial to the entire request based annotation framework since the name quality plays a fundamental figure the last annotation execution.

\section{E. Comparative Face Retrival}

In this step we depict the methodology of face annotation in the midst of the test stage. In particular, given a request facial 
picture for annotation, we first direct a tantamount face recuperation technique to chase down a subset of most similar stands up to (conventionally best $\mathrm{K}$ relative face tests) from the already recorded facial database. With the course of action of top K relative face cases recuperated from the database, the accompanying step is to remark the facial picture with a name (or a subset of marks) by using a bigger part voting approach that unites the game plan of names associated with these top $\mathrm{K}$ practically identical face delineations.

\section{F. Random Walks With Restarts:}

For non-Web pictures that are absence of printed data, an importance model-based calculation utilizing visual data is utilized to choose the hopeful annotations. At that point, the annotations are refined by re-positioning the hopeful annotations and saving the top ones. To determine the issues of, a calculation utilizing Random Walk with Restarts (RWR) is proposed to re-rank the hopeful annotations. The calculation not just uses the corpus data by characterizing a co-event based likeness, additionally influences (builds) the positioning and certainty data of unique annotations.

\section{ANALYSIS}

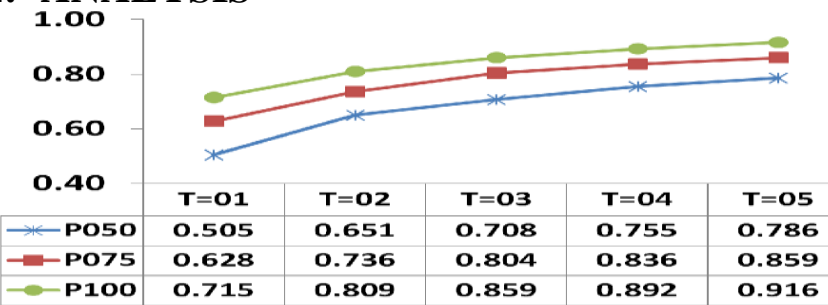

Fig. 2: The annotation performance on three different databases

As exhibited in above Fig. 2 the annotation execution on three one of a kind databases, which have various amounts of pictures per singular specifically, P050, P075, and P100 mean the databases have the primary 50,75 , and 100 recuperation pictures for every person, independently.

\section{CONCLUSION}

This paper tended to the face annotation issue in online networking. Without a doubt, late client studies report that individuals want to sort out their photographs as per who shows up in their photographs (e.g., relatives or companions). The fundamental point of this paper is to maintain a strategic distance from the duplication of names by utilizing random walk (RW) with restarts based semi-directed learning strategy. Utilizing Random walk, this framework re-positions the hopeful annotations, in which both the web data and certainty scores of unique annotations are fortified. RW tackles the general substance based face annotation issue utilizing the pursuit based technique. The investigation additionally shows promising results.

\section{REFERENCES}

[1] S. Satoh, Y. Nakamura, and T. Kanade, "Name-It: Naming and Detecting Faces in News Videos," IEEE Multimedia, vol. 6, no.1, pp. 22-35, Jan.-Mar. 1999.

[2] A.W.M. Smeulders, M. Worring, S. Santini, A. Gupta, and R. Jain, "Content-Based Image Retrieval at the End of the Early Years," IEEE Trans. Pattern Analysis and
Machine Intelligence, vol. 22, no. 12, pp. 1349-1380, Dec. 2000.

[3] S.C.H. Hoi, R. Jin, J. Zhu, and M.R. Lyu, "SemiSupervised SVM Batch Mode Active Learning with Applications to Image Retrieval," ACM Trans. Information Systems, vol. 27, pp. 1-29, 2009.

[4] T.L. Berg, A.C. Berg, J. Edwards, and D. Forsyth, "Who's in the Picture," Proc. Neural Information Processing Systems Conf.(NIPS),2005.

[5] D. Ozkan and P. Duygulu, "A Graph Based Approach for Naming Faces in News Photos," Proc. IEEE CS Conf. Computer Vision and Pattern Recognition (CVPR), pp. 1477-1482, 2006. [6] M. Guillaumin, T. Mensink, J. Verbeek, and [6] C. Schmid, "Automatic Face Naming with Caption-Based Supervision," Proc. IEEE Conf. Computer Vision and Pattern Recognition (CVPR), 2008.

[7] M. Guillaumin, T. Mensink, J. Verbeek, and C. Schmid, "Face Recognition from Caption-Based Supervision," Int'l J. Computer Vision, vol. 96, pp. 64-82, 2011.

[8] T. Mensink and J.J. Verbeek, "Improving People Search Using Query Expansions," Proc. 10th European Conf. Computer Vision (ECCV), vol. 2, pp. 86-99, 2008.

[9] T.L. Berg, A.C. Berg, J. Edwards, M. Maire, R. White, Y.W. Teh, E.G. Learned-Miller, and D.A. Forsyth, "Names and Faces in the News," Proc. IEEE CS Conf. Computer Vision and Pattern Recognition (CVPR), pp. 848-854, 2004.

[10] M. Zhao, J. Yagnik, H. Adam, and D. Bau, "Large Scale Learning and Recognition of Faces in Web Videos," Proc. IEEE Eighth Int'l Conf. Automatic Face and Gesture Recognition (FG), pp. 1-7, 2008.

[11] Z. Wu, Q. Ke, J. Sun, and H.-Y. Shum, "Scalable Face Image Retrieval with Identity-Based Quantization and Multi-Reference Re-Ranking," Proc. IEEE Conf. Computer Vision and Pattern Recognition (CVPR), pp. 3469-3476, 2010.

[12] D. Wang, S.C.H. Hoi, Y. He, and J. Zhu, "RetrievalBased Face Annotation by Weak Label Regularized Local Coordinate Coding," Proc. 19th ACM Int'l Conf. Multimedia (Multimedia), pp. 353-362, 2011.

[13] D. Wang, S.C.H. Hoi, and Y. He, "A Unified Learning Framework for Auto Face Annotation by Mining Web Facial Images," Proc. 21st ACM Int'l Conf. Information and Knowledge Management (CIKM), pp. 1392-1401, 2012.

[14] Dayong Wang, Steven C.H. Hoi, Ying He, and Jianke Zhu," Mining Weakly Labeled Web Facial Images for Search-Based Face Annotation" IEEE Transactions on Knowledge and Data Engineering, vol. 26, no. 1, January 2014

[15] W. Dong, Z. Wang, W. Josephson, M. Charikar, and K. Li, "Modeling LSH for Performance Tuning," Proc. 17th ACM Conf. Information and Knowledge Management (CIKM), pp. 669-678, 2008. 